\title{
Sociologie et morale sociale
}

\section{Christian Lalive d'Epinay}

\section{OpenEdition \\ Journals}

Édition électronique

URL : http://journals.openedition.org/ress/551

DOI : 10.4000/ress.551

ISSN : 1663-4446

\section{Éditeur}

Librairie Droz

\section{Édition imprimée}

Date de publication : 1 septembre 2003

Pagination : 181-193

ISBN : 2-600-00878-0

ISSN : 0048-8046

Référence électronique

Christian Lalive d'Epinay, "Sociologie et morale sociale », Revue européenne des sciences sociales [En ligne], XLI-126 | 2003, mis en ligne le 30 novembre 2009, consulté le 23 avril 2019. URL : http:// journals.openedition.org/ress/551 ; DOI : 10.4000/ress.551 


\section{Christian LALIVE d'EPINAY}

\section{SOCIOLOGIE ET MORALE SOCIALE}

Le thème que Jacques Coenen-Huther propose à notre réflexion m'amène à faire une pause au bord du chemin. Back to basics. La sociologie est-elle, par nature, relativiste? Peut-on, de la sociologie, discipline analytique, tirer des enseignements normatifs, des principes-guides? Du coup je m'interroge: comment, sur la base de quels axiomes, pratiquai-je ma science? Quels liens, ou quelles ruptures, établis-je entre ma science et ma vie? Que m'enseignent ma science et ma recherche sur la société d'aujourd'hui et la marche du monde? Et en quoi cela m'interpelle-t-il?

Ces questions étaient le pain quotidien qui nourrissait la réflexion des pères fondateurs. Sous l'effet de la professionnalisation de la discipline, avec son découpage en une nuée sans cesse croissante de spécialisations et de sous-spécialisations, voici que s'opère la rupture entre les «chercheurs» et les «penseurs » (rares, donc précieux). Elle conduit les premiers, en ingénieurs du social qu'ils sont, à abandonner la question des fondements aux seconds; englués dans leurs terrains, ils trouvent mais ne pensent plus guère. Les «penseurs», de leur côté, savent sans même ressentir le besoin de chercher, dans la clarté si lumineuse de leur cerveau et de leur bibliothèque qu'elle leur épargne de s'astreindre au corps à corps avec la réalité qui est le propre de la recherche empirique.

Jacques nous invite à revoir cela. A oser dire ce que l'on sait, ou tout au moins ce que l'on croit savoir, tant il est vrai que s'interroger sur les fondements revient à considérer les axiomes (prémisses tenues pour vraies) de sa démarche. D'ailleurs, à ce niveau premier de la construction du savoir, où est la frontière entre savoir et croyance?

Pour ma part, s'il me faut me classer entre «chercheurs» et «penseurs», je choisis le camp des laboureurs du réel, mais il se trouve que le fait de chercher, que je trouve ou ne trouve pas importe peu car ici le labour est ici aussi important que la récolte, me donne à penser, à penser à ce que je fais et à ce que je sais. Et c'est ainsi que l'invitation de Jacques me donne l'occasion, une nouvelle fois, de faire le point sur quelques questions fondamentales, et de l'exposer sans me dissimuler derrière l'autorité ou la référence ${ }^{1}$.

Au cours de ces pages, mon objectif sera de dégager quelques axiomes de la démarche sociologique, de les passer au crible de ma méditation, et de me

Ma démarche prenant aujourd'hui la forme d'une «méditation sociologique», je renonce cette fois à l'usage de la référence bibliographique, et ne préciserai mes sources que lorsque j'emprunte en dehors du stock généralement partagé des sciences sociales. 
demander s'il en découle des normes morales qui peuvent - ou peut-être doivent - être posées en universaux.

\section{DU PARADOXE FONDATEUR DE LA SOCIOLOGIE}

\section{Le postulat universaliste}

Ce paradoxe peut être énoncé ainsi: au fondement de la démarche sociologique gît un universel, à savoir le postulat d'unicité du genre humain. Mais en même temps, de cet universel, de ce qui fait l'unicité du genre humain, découle la diversité des cultures et des sociétés, et donc leur caractère relatif. Relatif à une histoire, à un temps et un espace, à une praxis humaine. Le paradoxe tient donc dans l'affirmation conjointe de l'universel et du divers, et dans l'ancrage de la diversité dans l'universel.

Nous posons l'unicité de l'espèce humaine en axiome. Est-ce une croyance, est-ce un savoir? De fait, nous recevons cet axiome des biologistes et, à ce jour, nous pouvons tenir pour démontré ou, si l'on préfère, pour «non falsifié » l'affirmation d'un équipement biologique de base, partagé par tous les individus de l'espèce humaine, cela quel qu'ait pu être l'impact de l'environnement et des conditions de vie sur certains groupes.

L'axiome universaliste est présent, plus ou moins explicitement, dans l'œuvre de tous ceux que nous reconnaissons comme pères fondateurs. Certes on s'est interrogé avec Lévy-Bruhl sur la «mentalité primitive» comme forme distincte de la pensée rationnelle, mais l'idée d'un fonctionnement génétiquement différent est vite écartée. Au profit cependant, dira-t-on, d'un évolutionnisme qui légitime de classer les peuples et les cultures, d'inférieur à supérieur, de primitif à évolué. Mais l'hypothèse évolutionniste, à savoir que la dynamique des sociétés et des rencontres de sociétés produisent des changements, que certains d'entre eux soient de nature cumulative ou s'imposent comme irréversibles, que ces changements s'organisent selon des schèmes exprimant d'après certains quelque grandiose loi de l'histoire ou, plus modestement pour d'autres, parmi lesquels je me compte, un «procès de civilisation", voilà qui n'est en rien incompatible avec l'axiome universaliste et qui présente une ligne de recherche et d'interrogation conséquente avec l'affirmation de la diversité des cultures et des sociétés.

Retenons à ce stade cette affirmation universaliste de la sociologie et, puisque ce colloque se tient à Genève, signalons que même dans ce petit pays épargné par les guerres du $\mathrm{XX}^{\mathrm{e}}$ siècle et par la folie brune et noire, les attaques contre la sociologie comme science de l'universel ont été rudes des années 1920 jusqu'après la seconde guerre mondiale; selon Zürcher (1995), notre discipline n'a été préservée qu'à l'Université de Genève. Cette exception est le fruit de deux raisons principales: l'ancienneté de l'enracinement universitaire de la sociologie (la chaire de sociologie remonte à la fin du $\mathrm{XIX}^{\mathrm{e}}$ siècle), la présence et les échanges avec les institutions internationales. Ailleurs lui ont été substituées des pseudo-sciences humaines fondées moins sur le sang que sur le sol, avec à Zurich par exemple la création d'un institut de nature eugéniste, et tout cela sur fond d'antisémitisme, certains professeurs juifs subissant d'ailleurs de véritables «Berufsverbot» (interdiction d'enseigner). C'est pendant ces décennies de cauchemar que Jean Piaget, 
qui fut ici le maître de plusieurs d'entre nous, a démonté les mécanismes de l'intelligence et les étapes de sa formation chez l'enfant, apportant ainsi une contribution d'importance au postulat universaliste de l'être humain, contribution majeure que le sociologue salue avant d'en critiquer le formalisme et le caractère anhistorique.

\section{L'implication morale du postulat universaliste}

Peut-on tirer un principe moral de cet universel qui est à la base des sciences de l'homme? Sa conséquence logique me paraît venir étayer l'intuition fondamentale des Droits de l'Homme: les hommes naissent égaux. Au-delà des différences qui les singularisent et des inégalités qui les hiérarchisent, ils sont fondamentalement égaux, donc égaux en droit.

On objectera ici que le postulat d'universalité comme que le principe égalitaire sont des idées qui ont émergé avec l'avènement des temps modernes au sein d'une aire de civilisation précise, et qu'ainsi ce sont des idées relatives. On peut aussi ajouter que l'idée égalitaire a une généalogie dans la très longue durée et que le fait égalitaire a été trahi aussitôt qu'il fut affirmé, par les révolutionnaires euxmêmes qui reconduisirent l'esclavage et qui sanctionnèrent l'inégalité citoyenne des sexes. Mais en même temps que nos sciences m'enseignent que l'égalité ne pouvait pas être pensée comme principe universel dans certaines cultures, elles me montrent également qu'au fur à mesure qu'elle s'affirme et se répand, elle est dans une société qui se mondialise une idée-force qui impose son évidence et remplit «d'une sorte de terreur religieuse» (selon la belle expression de Tocqueville dans l'introduction à De la Démocratie en Amérique) ceux qui tendent à lui résister de par leur éducation ou leur idiosyncrasie personnelle.

\section{De l'unicité du genre humain à la diversité des cultures et des sociétés}

L'unicité du genre humain est fondée dans le double constat de l'équipement (génétique) de base identique des individus de l'espèce d'une part, et de la similitude des problèmes auxquels ces individus sont toujours et partout confrontés, d'autre part. Comme l'ont bien saisi Marx et Engels, la survie de l'individu et de l'espèce constitue les contraintes les plus immédiates. Et c'est ici que l'équipement génétique de l'être humain présente une caractéristique intéressante, qui n'est pas l'exclusivité de cette espèce mais qui s'y présente comme tout particulièrement exigeante et développée, à savoir la pensée ou la raison, ou encore, comme il sied de dire dans nos disciplines, la réflexivité. Dès lors, et cette fois contrairement à Marx et Engels (cf. l'Idéologie allemande, mais pour leur défense rappelons qu'ils réagissaient contre l'idéalisme hégélien), la pensée et avec elle ses œuvres, la technique, le droit, l'art, la religion, etc. n'émerge pas une fois les besoins de base satisfaits, mais se présente comme la condition même de la satisfaction de ces besoins.

Dès lors, l'aventure humaine se profile en se diversifiant selon une dialectique permanente: un groupe humain, son environnement, son héritage culturel (son stock de savoirs et de croyances), les événements qui surgissent. Un autre groupe humain, la même dialectique fondamentale, mais une autre aventure et d'autres 
solutions. Au fil des générations, la codification de nouvelles réponses, et aussi l'abandon de réponses jugées mauvaises ou obsolètes (auxquelles, en ce qui concerne notre propre passé, nos musées d'ethnographie européenne offrent un cimetière). Les solutions apportées sont-elles vraiment radicalement singulières et différentes les unes des autres, ou s'agit-il de variations autour d'un thème commun? Voilà l'objet empirique d'une sociologie comparée et théorique d'une anthropologique générale, et à ce stade de l'inventaire, même pour ceux que l'entreprise structuraliste d'un Lévi-Strauss n'a pas convaincu, rien n'exclut d'écarter l'hypothèse d'une grammaire générale du social.

Constatons, à ce stade, le statut fondamental de la culture dans l'étude des sociétés humaines, ce lieu des savoirs et des croyances, des valeurs et des règles, avec d'un côté la problématique de la socialisation comme processus de transmission d'une partie plus ou moins large du stock culturel du groupe aux individus, d'une génération à l'autre, et de l'autre, celle de la production des savoirs et des croyances. Ce qu'on appelle la réflexivité n'est rien d'autre que cette capacité d'élaboration des problèmes posés par l'expérience du monde réel, une élaboration qui opère par référence au stock de connaissances, matrice de l'organisation réflexive de l'expérience. La culture est ainsi une exigence de la nature humaine en même temps qu'elle est un donné du monde des hommes.

\section{De la reconnaissance de l'altérité}

De cette diversité des cultures, fille paradoxale de l'universalité du genre humain, découle-t-il quelque autre universel moral? En bonne logique, si je pose le principe d'égalité des êtres humains, s'impose également, par voie de conséquence, la norme du respect de l'altérité, la reconnaissance de l'Autre comme différent et de son droit fondamental à la différence. Cette matrice qui l'a construit et qui le fait vivre, sa société et sa culture (ou encore, au sein d'une même société, la «niche» qui y fut la sienne), est différente de la mienne, mais intrinsèquement ni inférieure, ni supérieure. Et ainsi surgit ce qui est une hypothèse de base de la méthode dans les sciences sociales, et en même temps un principe recteur du rapport à l'autre, à savoir que ce qui me paraît absurde n'est rien d'autre qu'un comportement dont le sens m'échappe, que ce qui «instinctivement» (les guillemets sont là pour rappeler que ce qu'on appelle l'instinct n'est ici que le produit d'une socialisation) me paraît barbare est le fruit d'une culture que je méconnais. Là se trouvent, à mes yeux, tout l'intérêt et toute la noblesse de la démarche sociologique ou ethnologique: l'autre est un avatar de moi-même dont, sociologue ou ethnologue, je cherche à reconstruire l'histoire, génétique, sociale et culturelle. Ce qui, par détour (l'expression, on le sait, est de Balandier, mais l'idée se trouve chez Montaigne, et avant lui chez Socrate et sans doute quelques autres) m'apporte quelque lumière sur moi-même, comme avatar de l'Alter, cet universel. Cet autre n'est pas nécessairement Bororo, Persan, «primitif » Australien ou Mapuche du Cône sud américain, il est aussi l'ouvrier de chez Renault ou Flaubert, le vieillard dans un foyer, le cadre supérieur, ou encore l'adolescent d'aujourd'hui; et l'altérité est autant présente dans une institution comme le Conseil d'Etat français, IBM ou le PACS, que dans celle du potlach ou de la koula, du baptême chrétien, de la circoncision juive, ou encore dans les rituels d'anthropophagie ou d'excision. 
De la reconnaissance de la diversité et de la recherche d'une explication de l'altérité, s'en suit-il une indifférence morale, que tout serait également bon, désirable, juste, ou encore que tout serait également neutre? Ma réponse sera résolument négative, comme on peut déjà le pressentir de ce qui précède, mais à ce stade, contentons-nous de poser la question.

\section{HOLISME ET INDIVIDUALISME MÉTHODOLOGIQUES}

\section{Paradigmes antagoniques ou complémentaires?}

C'est alors que nous croisons un autre débat, qui depuis plusieurs décennies occupe de manière périodique et récurrente la scène sociologique française, celui du holisme et de l'individualisme méthodologiques. Autant le dire tout de go, depuis ma province genevoise, ce débat me remplit de perplexité tant il me paraît donner dans l'absurde. Quand je lis L. Dumont, je suis résolument «holiste»si par-là on entend qu'une civilisation ou une culture font « système » et, pour donner un exemple, par mes travaux sur la période contemporaine, je rejoins largement Ehrenberg en concluant qu'aujourd'hui l'individualisme n'est pas un choix individuel, mais un cadre culturel. Quand en revanche, je médite le dernier opus de Boudon ou encore (tout en ne prenant pas Boudon pour un tenant de l'interactionnisme symbolique) quand je reprends un ouvrage de Goffman, il me paraît évident que l'individu trouve de bonnes raisons à agir comme il le fait, qu'il élabore ses solutions et que, même enfermé dans une institution totale, il est doté d'une marge de liberté et d'action. De même, quand suivant par exemple Norbert Elias, je fais la sociogenèse d'une institution, je contemple l'enchevêtrement complexe des interactions de générations d'êtres humains dont cette institution résulte. Serais-je alors un sociologue sous influence, l'influence de sa dernière lecture en date? Examinons cela de plus près.

Le postulat du holisme méthodologique me paraît difficile à discuter, pour autant que ce que j'ai appelé plus haut système ne soit pas conçu comme clos toute culture est toujours inachevée et porteuse de tensions internes -, ni comme figé - les hommes y sont toujours à l'œuvre et l'histoire continue -, ni comme doté de forces - c'est, à bien lire Durkheim (Les Formes...), l'acte de croire qui rend plus fort, donc la relation de l'individu à la croyance, et non la croyance en soi. Ainsi donc, il faut rappeler qu'une culture n'est vivante que par les hommes qui la vivent, donc en animent des segments; en soi elle n'est que latence (pour parler comme Sorokin et Parsons), donc pur potentiel. Cette réserve essentielle faite, le paradigme du holisme me paraît être indispensable à notre pratique, à notre volonté de trouver l'intelligibilité de l'Alter. Comment entrer dans un système ou sous-système autre sans en acquérir quelques clés, le langage bien évidemment, paradigme d'un système jamais clos, jamais achevé, et qui n'est force que par l'usage que les hommes en font. Comment, jeune chercheur, auraisje pu «entrer» dans l'Amérique hispanique et le mouvement pentecôtistes chilien sans l'apprentissage d'une des principales langues vernaculaires, sans la compréhension de quelques autres clés de base, un rapport au temps assez différent de celui du jeune Suisse, une identité grinçante entre indianité et hispanitude et, en ce 
qui concerne plus particulièrement le sujet de mes travaux, des formes de solidarité rendant possible l'art de la survie dans un contexte menaçant.

De son côté, tout aussi indiscutable et foncièrement stimulant me paraît le postulat de l'individualisme méthodologique selon lequel l'action, les croyances, les attitudes des individus sont à la base de tout phénomène social, selon lequel tout acteur est placé face à des dilemmes, à des choix, donc devant une pluralité de possibles? Une pluralité, non mais sans limite. Contemplant ses contemporains et soi-même, Max Weber évoquait «la cage d'airain» que constituait l'ethos du travail pour l'Européen du début du $\mathrm{XX}^{\mathrm{e}}$ siècle. Lui, l'individualiste méthodologique, décrivait alors les bornes de la pensée et de l'action individuelle que marquait alors l'ethos d'une société organisée autour du travail industriel, de même, répétons-le, qu'Ehrenberg et d'autres décrivent le champ culturel, avec ses limites, des sociétés post-industrielles.

Le holisme s'impose pour autant que l'on affirme que la culture constitue un champ du possible pour ceux qui la vivent, et qu'on se souvienne, avec Tocqueville, que ce champ est beaucoup plus riche de possibilités que la plupart des hommes d'une période historique donnée ne l'imaginent. L'individualisme est incontournable pour autant qu'on précise qu'action, croyance et attitude ne sont possibles que grâce à «l'héritage», c'est-à-dire au fait que nous naissions dans une culture qui s'offre comme un formidable outil de pensée et d'action, mais qui, dans le même temps, leur fixe des limites. Evoquons ici la rencontre des Espagnols avec les Indiens, de Cortez avec l'Aztèque Moctezuma, ou un peu plus tard de Pizarro avec l'Inca Atahualpa. Pour les premiers, guidés par leur catholicisme, la question était de savoir si les Indiens étaient des hommes ou des animaux, ou encore quelque être intermédiaire, mais ils ne pouvaient être des dieux, puisqu'il n'y en a qu'un et qu'il est avec les Espagnols, et que si par hypothèse ce sont des hommes, de toute évidence il s'agit de l'espèce païenne de l'humanité. Pour les Aztèques comme pour les Incas, du fait cette fois de leurs propres mythes et croyances, le dilemme était de savoir si ces êtres issus de la mer étaient des dieux ou des hommes, avec l'exclusion de l'hypothèse d'une identité animale. Et si par hypothèse, ils sont d'espèce divine, alors leur résister semble bien vain (cf. sur ce thème, Léon-Portilla, 1959; Wachtel, 1971).

Evoquons encore le Robinson cher aux économistes classiques qui l'érigent en paradigme de l'individu isolé construisant son monde sur la base d'un comportement dit rationnel? Mais avec Robinson Crusoé, ou Daniel Defoe, c'est le parfait britannique du XVIII ${ }^{e}$ siècle naissant qui échoue dans l'Ile Juan Fernandez, porteur d'un savoir technique particulièrement efficace en situation de survie, mais aussi de la vision du monde britannique, donc hiérarchique et impériale. Souvenons-nous de la sortie de sa solitude: il dote d'un surnom - Vendredi - le sauvage qu'il sauve d'un funeste destin, mais au lieu de lui révéler le sien Robinson -, il lui demande de l'appeler au moyen d'un titre: «I likewise taught him to say Master, and let him know, that was to be my name».

Si l'on fait de la culture une totalité close, l'aventure humaine est récapitulée sous la forme d'une série d'entéléchies juxtaposées dans l'espace ou successives dans le temps. Les Pyrénées sont alors des barrières sans cols, et ceux qui par accident les franchissent doivent renaître ou se convertir (ce qui revient au même!). 
Tel est bien le risque que présente l'ethnologie classique, qui dans sa tentative de reconstruction des sociétés et des cultures de l'ailleurs, offre trop souvent au lecteur le tableau d'un système refermé sur lui-même et achevé. Dans sa démarche, elle dégage la structure et la cohérence, négligeant les dilemmes que posaient les solutions inventées, les impasses dans lesquelles elles débouchaient souvent. Ainsi réifie-t-elle ce qui n'est que reconstruction idéal-typique (une caricature donc, pour reprendre le terme de Weber) d'une réalité dynamique, close ou problématique, produit provisoire du bricolage et de l'inventivité de générations. Mais, puisque je viens de citer Weber, j'ajoute que pour ma part je ressens de la méfiance quand il s'exclame que toute culture est un «chef-d'œuvre». Quand bien même cette formule est admirable par le respect de l'altérité qu'elle exprime, elle prête à confusion car un chef-d'œuvre est une œuvre achevée, qui en aucun cas ne saurait être retouchée, dont la place est dans les musées car en vérité il n'y a de culture achevée que morte.

Cette conception ouverte de la culture, chantier permanent des hommes, n'enlève rien à son statut anthropologique de matrice du langage, du sens, du «possible» pour ces mêmes hommes, stock à partir duquel ils bricolent sans cesse dans leur volonté de rendre le monde et la vie intelligible. Et même dans une modernité imprégnée de la pensée scientifique, le réel résiste à l'entendement, les interrogations existentielles des hommes perdurent, et la science, qui ne dit rien de l'essentiel, ne détruit pas la croyance. Ironie des temps, voici Elisabeth Tessier docteur de l’Université René Descartes!

Holisme et individualisme méthodologiques ne me paraissent pas imposer un choix fondamental à la pensée sociologique, mais au contraire proposer les pôles paradigmatiques à nos disciplines «molles »; je ne les vois pas comme des théories qui revendiquent le monopole de la démarche explicative, mais comme des orientations de recherche que prend le chercheur pour explorer jusqu'où elles peuvent le mener et quelles en sont les limites, où s'en rencontrent les impasses. Dans cette perspective, l'un et l'autre me semblent indispensables à notre discipline.

Mais alors, peut-on tirer de l'un comme de l'autre, ou de l'un ou de l'autre, quelque universel d'ordre moral?

\section{De la finalité de la société...}

L'individualisme méthodologique m'enseigne que tout fait social comme tout segment culturel sont le produit d'une histoire et donc de l'activité humaine. Il m'interdit, dès lors, de croire que la culture serait dotée d'une vie propre, le lieu d'un génie ou encore d'un «esprit» (Geist), bref de la traiter comme entéléchie. L'ethnologue comme le sociologue constate la tendance séculaire de l'activité humaine à sacraliser la culture, d'appeler Dieu ce qui ne serait qu'une représentation «idéalisée» (Durkheim) ou «déformée» (Marx) de la société en vue de répondre à une quête de sens ou à une volonté de domination, le plus souvent aux deux à la fois. Mais la leçon des sciences sociales n'est-elle pas qu'il n'est de «garant meta-social» régulateur de l'ordre des hommes ni dans les cieux, ni dans l'histoire, ni dans les gènes? Si une tendance de l'activité des vivants, dans leur quête de sens et de puissance, consiste à sacraliser la culture en appelant dieu 
quelque forme idéalisée de la société (Durkheim), l'analyse conduite par les sciences sociales renvoie l'homme et ses œuvres à lui-même, dans la stricte immanence de son existence. Mais dans le même mouvement, ces sciences lui enseignent la raison d'être de la société et de la culture, conditions nécessaires de la vie humaine. Nous voici renvoyés au paradoxe initial. La société et la culture sont les moyens à la disposition des hommes - les seuls moyens mais quels formidables moyens ! - de déployer leur vie dans les limites du donné biologique, et de préserver celle de l'espèce humaine.

Dès lors, ne doit-on reconnaître dans le fait de permettre le déploiement de la vie humaine la finalité première et la raison d'être de toute société? On en conclut que la légitimité morale d'un ordre social dépend de la mesure dans laquelle il satisfait à l'exigence de vie. J'évoquais plus haut la question classique de savoir dans quelle mesure le relativisme sociologique, à savoir la reconnaissance et le respect de l'Altérité conduiraient, sur le plan moral, au relativisme ou à l'indifférence. Ne peut-on proposer maintenant un critère d'évaluation pragmatique, à savoir qu'une institution sociale et culturelle peut être jugée en fonction de la contribution qu'elle apporte à l'entreprise collective de production du respect du droit égalitaire à la vie, dans un contexte socio-historique donné?

Recourons à un des exemples classiques de l'anthropologie: si les individus de toutes les espèces animales vieillissent, ce n'est qu'au sein des sociétés humaines, et dans les limites de leurs ressources, que les individus peuvent devenir vieux et avoir droit de cité? Ce n'est là qu'un indicateur, et pas le plus important, de la qualité d'un lieu social qui aménage la vie individuelle et la vie collective. Dans le contexte contemporain d'une société-monde, cette approche ne donne-t-elle pas un éclairage spécifique à la réflexion sur ces pratiques discutées et pourtant porteuses d'identité et d'appartenance que sont les rituels d'enterrement ou même d'excision?

\section{... et de la dette de l'individu}

En réciprocité de perspective, quel enseignement tirer du holisme? Que la vie humaine n'est possible qu'en société (et que même la survie de l'individu isolé n'est possible parce qu'il porte en lui des segments de la culture d'une société, comme on l'a vu à l'exemple de Robinson). Que depuis que l'homme est homme, l'individu humain naît au sein d'un ensemble socioculturel, et que s'il parvient en vie à l'état d'adulte autonome - à l'âge dit de «raison»-, c'est à cette société qu'il le doit, par le truchement d'un ensemble de groupes et d'institutions intermédiaires qui l'ont porté jusque-là? Dès lors, ne peut-on poser le principe de la dette de l'individu envers la collectivité à qui il doit (partie de) sa vie?

La civilisation chrétienne dénonçait le péché et appelait à la repentance, donc à la soumission à l'ordre divin et à son expression terrestre; l'Ancien Régime enseignait le devoir, en particulier celui de tenir son rang avec son cortège de contraintes. Avec le grand mouvement d'émancipation du XVIII ${ }^{\mathrm{e}}$ siècle, on s'est mis à affirmer les droits fondamentaux de l'individu. Aujourd'hui, prisonniers de la «cage d'airain» de l'individualisme, mes contemporains sont allergiques à l'idée que chacun de nous aurait une dette envers la société et font une poussée d'urticaire chaque fois qu'elle est évoquée. Mais développons l'idée. Au fondement de la citoyenneté moderne, n'y a-t-il pas l'exigence de la formation d'une 
conscience civique? Les croyances surplombantes se sont dissoutes dans l'éther, les Grands Récits sont partis en lambeaux; que reste-il que nous partagions, sinon cette certitude élémentaire, fondée sur l'expérience empirique de chacun: je vis ! Et je sais que je vais mourir! A ce stade d'un long parcours historique riche en élaborations fantasmagoriques destinées à permettre aux hommes de ruser avec la mort, nous voilà renvoyés à cette expérience brute qui définit la condition humaine depuis son origine, le constat de la vie, dans sa précarité, avec la conscience de la mort. Voilà que les Grands Récits ont perdu beaucoup de leur pouvoir de conviction, et les institutions qui les imposaient dépouillées de leur pouvoir coercitif. La croyance est aujourd'hui, on le sait, une affaire privée. Mais est-ce à dire que tout grand récit serait à jamais éteint? Pour ma part, il me paraît voir s'en développer un, qui paraît historiquement unique en ce qu'il se développe sans recours à l'idée de transcendance ni même de déterminisme, et qu'il est fondé sur l'expérience quotidienne de l'unique certitude empirique que les êtres humains peuvent partager, à savoir le fameux «je vis», ce «geste de l'homme éveillé » dont Groethuysen (1977) a retracé l'origine chez le bourgeois du XVII ${ }^{\mathrm{e}}$ siècle. Cette croyance ne serait-elle pas en train de produire un nouveau Grand Récit?

C'est Claude Lévi-Strauss qui disait que dans les sociétés modernes l'histoire s'était substituée au mythe (ce qu'il vient de répéter au cours d'un émouvant entretien télévisé (Campus, France 2, octobre 2002), et Michel Serres (2002) prolonge l'idée en suggérant que l'histoire de l'univers et de la vie, comme synthèse précaire de l'état des connaissances scientifiques, fonderait aujourd'hui un nouveau Grand Récit unitaire et universel, celui de la vie.

Le risque est ici celui d'une sacralisation de l'immanence; ayant perdu l'espoir de l'éternité, l'homme relance la quête de l'immortalité et des nouvelles fontaines de Jouvence alimentées par les développements des neurosciences. Mais laissons-cela ici (pour des développements, cf. Lalive d'Epinay, 2000). De ce constat partagé: «je vis », dérive le constat de la dette de reconnaissance envers ce qui a permis ma vie, et donc ce contrat de restitution qui fonde la citoyenneté, à savoir l'engagement à participer à cette entreprise de vie qu'est la construction permanente de la société.

L'idée de la dette de l'individu envers la collectivité ne doit pas être confondue avec le principe de soumission à un ordre social quelconque, posé comme ordre sacré ou découlant d'une volonté sacrée. La dette de citoyenneté ne se comprend qu'en référence à la raison d'être de la société: assurer la vie en aménageant la vie collective. C'est ainsi d'un engagement critique et exigeant dont il est question, un engagement qui appelle pour être efficace le travail analytique, critique et démystificateur, des sciences sociales: la vocation du sociologue et celle du citoyen convergent, sans se confondre, dans l'idéal d'un plus d'humanité, donc d'un plus de vie et surtout d'une vie de meilleure qualité pour les hommes et les femmes.

\section{La société aujourd'hui}

Reste à s'entendre sur ce que désigne, aujourd'hui, la notion de société. La conscience de la mondialisation présente cet avantage qu'elle rend évident que la société et son organisation ne peuvent aujourd'hui qu'être pensées à l'échelle 
de la planète. Distinguons ici le discours des pouvoirs économiques sur la mondialisation (globalisation) qui veulent faire passer une vague mise à jour du libéralisme pour la nouvelle et définitive loi de l'histoire, du processus de mondialisation, un processus qui me paraît aussi inexorable que celui de l'égalisation des conditions paraissait à Tocqueville, celui-là comme celui-ci s'inscrivant dans une marche presque millénaire. La mondialisation est en germe sur le plan culturel depuis que l'homme s'est doté d'un outil (la quantification et la mathématique) lui permettant d'étudier et de penser l'univers de manière unifiée, elle est en marche depuis que Magellan ou du moins certains de ses compagnons, eurent bouclé la première circumnavigation; du voilier à la microinformatique, un processus accéléré de rétrécissement de l'espace-temps planétaire.

On peut avoir la nostalgie d'un univers diversifié, dans lequel les Pyrénées déjà séparaient des mondes culturels, dans lequel des civilisations cohabitaient en s'ignorant, ne s'entrouvrant qu'à d'intrépides passeurs habités par l'esprit d'aventure et par la recherche du gain. Mais la mondialisation est un fait social, extérieur aux individus (on pourrait ajouter aux sociétés et aux cultures de notre planète), général, et contraignant. Bien entendu, elle a un centre et des périphéries; elle s'impose par la force physique en créant de nouvelles dominations, comme par la force de conviction en créant des alliances d'intérêt. Ce processus unifie en diffusant des techniques et des croyances (dont l'idée égalitaire et démocratique), et dans son mouvement unificateur il détruit. Il détruit des cultures, il dilapide des ressources, il massacre des enfants, des femmes et des hommes.

Il détruit des cultures, c'est-à-dire des formules de lien social. Mais, je l'ai dit, une culture n'a rien de sacré et dès lors, la mort de cultures qui ne sont plus à même de répondre à leur finalité ne me paraît pas être le vrai problème, qui est celui la mort d'êtres humains que ces cultures faisaient vivre et pour qui elles disaient le sens.

Il dilapide, détruit des ressources. Des ressources aussi fondamentales que la forêt, l'air, etc., ces ressources même qui ont fait que, voici quelques milliards d'années, l'aventure de la vie a commencé sur cette planète. Voilà qui me paraît beaucoup plus préoccupant.

Enfin, cet avènement d'une «société-monde» (Mercure, 2001) détruit des hommes, par millions, chaque année, par les famines et les épidémies qu'il provoque, alors que les moyens de les juguler existent et sont à disposition (voir sur ce point le rapport récent [oct. 2002] de la FAO sur la sous-alimentation). Un anthropologue et médecin spécialiste des maladies infectieuses, Paul Farmer (2001), a démonté les mécanismes de la violence structurelle de la mondialisation et de ses acteurs économiques lors d'une magistrale leçon inaugurale au Collège de France. Farmer, qui vit et pratique la médecine une partie de l'année à Haïti, analyse comment «la distribution du sida et de la tuberculose est donnée historiquement et modelée économiquement», comment aussi, dans le procès de mondialisation, «la souffrance est réduite au silence ou franchement escamotée.» $\mathrm{Si}$ respectable que soit la mobilisation en faveur de cette femme nigérienne menacée de lapidation pour crime d'adultère, celle-ci me paraît douteuse quand elle sert d'alibi à la chape de silence qui recouvre les génocides en cours causés par la violence structurelle de la mondialisation. Dans quelle mesure, depuis 
l'effroyable attentat du 11 septembre $2001^{2}$, l'Islam et «l'islamisme» ne sont-ils pas érigés en épouvantail par un Occident soucieux d'escamoter ses propres crimes contre la vie?

On en est là. Englué dans le paradoxe d'une mondialisation qui diffuse les droits de l'homme en les piétinant et en affamant une partie de l'humanité. Comment, dans le contexte de mondialisation, affirmer l'égalité fondamentale des êtres humains, leur même droit à la vie d'un côté, et la reconnaissance de l'altérité et la cohabitation avec elle de l'autre? Comment contribuer à l'édification d'une société-monde répondant à sa raison d'être, alors que l'édification en cours est le fait d'architectes et de constructeurs aux services d'intérêts aussi précis que limités? L'entreprise paraît démesurée; elle l'est assurément à l'échelle de l'individu, et pourtant, pour le sociologue que je suis, cette dette de citoyenneté que j'évoquais prend la forme d'un impératif moral.

\section{CONCLUSION}

Récapitulons ici notre navigation entre sociologie et morale en longitude, universalisme et relativisme en latitude.

Il m'est apparu qu'au fondement de la démarche sociologique se trouve le constat d'un universel, l'être humain, qui par nature produit de la culture et trouve dans la culture son propre achèvement en tant qu'être social. Le destin de l'être humain réside ainsi dans une unité d'espèce, et une diversité de réalisations, les sociétés-cultures, ces dernières m'étant apparues comme les conditions de possibilité du développement de la vie humaine. Si l'on passe du registre de la connaissance à celui de la morale, du constat de l'universalité de l'être humain découle le principe de l'égalité des êtres humains, et donc de leur égalité de droit, en particulier à la vie, et de celui de la multiplicité des formations socioculturelles, celui du respect fondamental devant la diversité.

A ce stade j'ai croisé le débat du holisme et de l'individualisme méthodologiques, paradigmes que pour ma part je renvoie dos à dos ou, plus précisément, qui m'apparaissent comme deux propositions qui, loin de s'exclure, s'appellent l'une l'autre pour baliser le champ de l'activité sociologique et anthropologique. Du holisme je retiens que la culture est le stock des ressources dont dispose une société pour aménager la vie, et qu'elle contient les clés de lisibilité du comportement des membres de cette société. De l'individualisme je conclus que cette culture est le produit dynamique, ouvert, toujours en travail et donc toujours inachevé de l'activité des hommes, et que loin d'être une fin en soi, elle est le moyen de la vie sociale.

De l'individualisme méthodologique je tire que la société et la culture ne sont pas des fins en soi, mais que leur finalité morale, leur raison d'être sont de permettre le déploiement de la vie humaine, et que c'est à cette aune qu'elles doivent être évaluées, ainsi que toutes les institutions socioculturelles. Par

Par coïncidence, c'est aussi un mardi 11 septembre que voici bientôt trente ans, en 1973, a eu lieu au Chili un coup d'Etat militaire mettant bas un régime démocratique et entraînant l'assassinat de plus de 10000 personnes, le plus souvent dans des circonstances atroces. 
ailleurs, j'ai cru pouvoir constater qu'en ce grand soir des Récits mythiques, il en est un qui se développe en passant relativement inaperçu, le Récit de la Vie, ancré dans l'immanence de l'expérience quotidienne du «je vis». Cette croyance dans «la vie» ainsi que le déploiement de son grand récit ne sont pas sans risques, mais en ce qu'elle repose sur une expérience partagée, elle me paraît pouvoir fonder une morale sociale, tant il est vrai que l'affirmation «les hommes naissent égaux » contient dans son libellé même l'affirmation de la vie («naissent ») comme fondement de l'égalité, et donc la reconnaissance du droit à la vie comme droit universel.

Du holisme, je retiens que la société et la culture sont les conditions de la vie humaine, en ce sens, comme le disent les ethnologues, la culture fait partie de la nature humaine, et j'en conclus qu'un principe moral découle de la dette initiale de l'individu envers la société à laquelle il doit de vivre. Cette dette fonde le devoir de citoyenneté, qui n'est pas un devoir d'obéissance envers un ordre social donné, mais une exigence d'engagement critique dans la participation à la construction d'une société qui réponde à sa raison d'être: l'affirmation de la vie humaine.

Ce cheminement m'a enfin conduit à m'interroger sur la société à l'ère de la mondialisation, et à mettre en lumière que les problèmes les plus urgents sont aujourd'hui moins ceux que posent certains comportements de représentants de cultures «autres» (quelle que soit la visibilité médiatique dont ils sont l'objet), que la violence structurelle découlant des conditions actuelles de réalisation de la mondialisation (quel que soit l'escamotage médiatique et politique dont elles bénéficient).

Comment infléchir une société-monde qui s'édifie en répondant à une logique d'intérêts économiques en une société-monde organisée autour du droit à la vie? La question est citoyenne, au sens qu'elle est le problème de chacun, mais la vocation - la contribution - spécifique des sciences sociales n'est-elle pas, non seulement de se livrer à l'analyse critique donc rigoureuse des processus en cours, mais aussi de s'efforcer de tirer les leçons de l'aventure humaine pour une morale sociale?

Département de sociologie

Université de Genève

\section{RÉFÉRENCES BIBLIOGRAPHIQUES}

Farmer, P. (2001), «Une réalité horriblement intéressante» in: Le Monde, 11-12 novembre 2001.

Groethuysen, B. (1977 [1927]), Origines de l'Esprit bourgeois en France, Paris, Gallimard.

Lalive d'Epinay, C. (2000), «Le XXI' siècle ou le siècle des anthropoïdes?», in: J.-P. Fragnière, Y. Fricker et J. Kellerhals (Eds), La vérité est multiple. Essais de sociologie, Lausanne, Editions Réalités Sociales, pp. 95-103.

Léon-Portilla, M. (1957), La visión de los vencidos, Relaciones indígenas de la Conquista, Mexico, 1971.

Mercure D. (sous la dir. de), (2001), Une société-monde? Les dynamiques sociales de la mondialisation. (Actes du XVI congrès de l'AISLF, Québec, 2000), Québec et Bruxelles, Les Presses de l’Université Laval et De Boeck Université. 
Serre, M. (2002), «L'humanisme universel qui vient», in: Le Monde, 5 juillet 2002.

Wachtel, N. (1971), La vision des vaincus, Paris, Gallimard.

Zürcher, M. (1995), Unterbrochene Tradition: die Anfänge der Soziologie in der Schweiz, Zurich, Chronos Verlag, octobre 2002. 